\title{
THE ROLE OF THE HUMAN PERFORMANCE LABORATORY
}

\author{
H. THOMASON, M.Sc., Ph.D.
}

\section{Human Performance Laboratory, University of Salford *}

Over the last decade coaches and their charges have become more aware of the facilities that are available in some scientific establishments in this country which seemingly could be useful to them in their efforts to improve performance. The assessment of athletes in Human Performance Laboratories has now become more widespread. However, some physiologists have been guilty of using these athletes as guinea-pigs and providing little information that is of use to them in their quest for performance improvements.

A. V. Hill did the pioneer work in exercise physiology and when he was asked the question why he did it and would the findings be of use to athletes, he said "No! the reason we do this work is because it is fun" - at least he was honest! As long ago as 1964 a famous athlete's coach said to us before we presented some of our initial findings - "Look, all I want to know from you people is, how can I make him run faster and if he is not improving, why not?". I think the same is true today. Most coaches see the physiological assessment of their charges in laboratories such as ours at Salford as some magic elixir. They really don't understand what we are trying to do, the limits of what we can do, nor what value our findings could be to them.

Without going into too much detail, what we attempt to do is to assess the physiological efficiency of a subject (who is well-conditioned to the laboratory task) when performing known amounts of physical work. The work tasks must be specific to the event or events in which the subjects compete. That is, to try to go some way towards simulating the work demands of the subject's actual event in the laboratory work task, i.e. runners use treadmills, cyclists use ergometers. We attempt to build up profiles describing the subject's efficiency to perform work at various levels of intensity and duration. This study is in its infancy as far as we are concerned and, with the assistance of a grant of money from a Northern Insurance Company, $t$ we are following eight good, young athletes throughout the next four years.

Efficiency in the laboratory is seen as both actual

* Present address: Dept. of Physical Education and Sports Science, University of Loughborough, Leicestershire

†Grant support from Provincial Insurance Co. Ltd., Kendal. work done and the body's physiological response to it: i.e. the ability to handle the work. This information is $\overline{\bar{n}}$. then equated with similar work on the track. Eacho athlete is regarded as an individual study case and, as such, the individual's coach, the athlete and ourselvess will meet frequently to discuss the progress of the worko and any application of the findings to their training. $A-$ one-off assessment which produces a list of data about an athlete's ability to perform a battery of tests on one्D. day is of little value in the context of the work we are? doing.

Hopefully, we will eventually be able to describe the performance potential of an athlete - but this does not mean that we can actually describe his true potential aso seen by his performances in race conditions. Laboratory. tests of performance are limited in this and coaches andes athletes should be made aware of this fact from the outset.

The President of the American College of Sports Medicine recently delivered a paper on this subjexte entitled 'Science and the future of Sport'. 'What he said. is very relevant to our own situation in this country.

He emphasised that science has a definite opportunity to make an input into the pursuit of excellence. Sports scientists, in particular, with their dual training should be involved in the preparation of athletes. They must however, not fail to relate their findings to the athlete's problem in a manner that can be understood by all. $\mathrm{He}_{-}$ has a commitment to educate all concerned with the athlete. The athlete must not be used as a guinea pig but should derive some direct benefit from his participation? in a scientific study. This is not always easy to do but if must, nevertheless, be attempted.

However, one must not overlook the fact that some basic exercise research is needed to provide the fundamental knowledge and methodology necessary to answers the practical questions. Therefore, all should have a part to play in the development of an athlete's full potentiaE. but, equally, they must realise fully the limitations of o their gained knowledge in the context of improving this performance. If this is borne in mind the role of the Human Performance Laboratory, in the training of $w$ athletes in the pursuit of performance improvement, will be enhanced.

\section{REFERENCE}

1 Costill, D. L. 'Science and the future of Sport': Sports Medicine Bulletin of the American College of Sport疋 Medicine, April, 1977. 